\title{
Thermal Noise and the Incessant Vibration of the Outer Hair Cells in the Cochlea
}

\author{
W. FRITZE* and M. STEURER \\ ENT Department, University of Vienna Medical School, Vienna, Austria
}

(Received in final form 5 December 1997)

\begin{abstract}
The continual exposure of outer hair cells (OHCs) to thermal noise causes vibrations in resonant frequency. As these vibrations are backprojected, they should be recordable as audiofrequencies in the outer ear canal. But even though they are likely to be amplified in some areas by clustering in terms of the chaos theory, they cannot be picked up in the outer ear canal by currently available recording technologies. Conditions change in the presence of pathology, e.g. loss of OHCs and fibrous replacement: Clusters grow in size and amplitudes become larger so that the vibrations can be picked up as spontaneous otoacoustic emissions (SOAEs) in the outer ear canal. Efforts are needed to demonstrate the presence of physiological OHC vibrations (emission by incessant vibration, EIV) by processing auditory recordings with statistical methods.
\end{abstract}

Keywords: Inner ear function, Oto-acoustic emissions, Outer hair cells, Resonant vibration, Chaos theory, Emission by incessant vibration

\section{THERMAL NOISE AND RESONANCE}

There is evidence suggesting that events such as those recorded in quantum physics are bound to affect hearing (Bialek, 1985; Peat, 1987), because the amplitude of noise perceivable to the human ear is less than the diameter of a hydrogen atom. But as molecular motion is indeterminate, its action on the OHCs is likely to vary continually.

Any object capable of vibrating has its own resonant frequency. It is made to vibrate at the resonant frequency by random stimuli including thermal noise. It would be logical to assume that the resonant frequency is identical with the audiofrequencies corresponding to a given site along the basilar membrane. This would imply that it would have to change by 3 decimal points along the length of the basilar membrane. But as the anatomy does not change to such an extent along the basilar membrane, this is very unlikely. In addition, a vibration of no more than $20 \mathrm{~Hz}$ would also be quite unlikely for so small a structure.

\footnotetext{
* Corresponding author. Allgemeines Krankenhaus, Währinger Gürtel 18, A-1090, Vienna, Austria. Tel.: +43 1404005610. Fax: + 43140400 3332. E-mail: hno@uniw.ac.at.
} 
What is much more likely is that the resonant frequency is substantially higher than the audiofrequencies (Ohyama et al., 1985; Fritze, 1996). (In bats resonant frequencies are known to be very high; Kössl, 1994; Henson et al., 1990).

\section{THRESHOLD MICROSTRUCTURE}

If this were so, audiofrequencies and resonant frequencies should interact, i.e. there should be mathematical ratios of these 2 frequencies at which vibrations are either dampened or escalated. Take the example of a vibrating metal plate steadily pounded by a hammer. By choosing an appropriate pounding pattern the vibrations of the plate can either be intensified or almost stopped.

Assuming that the threshold microstructure (Elliot, 1958; Cohen, 1982; Long and Tubis, 1988) reflects this process, the resonant frequency at the site of the basilar membrane to which a $1 \mathrm{kHz}$ tone is projected would be roughly $25 \mathrm{kHz}$, as simulations indicate. For a $2 \mathrm{kHz}$ tone the resonant frequency at the appropriate site of the basilar membrane would be roughly $40 \mathrm{kHz}$.

\section{SPONTANEOUS OTO-ACOUSTIC EMISSIONS}

Another point is that it is difficult to understand without postulating incessant $\mathrm{OHC}$ vibrations how known SOAEs are generated. By their current definition SOAEs are sequences of sinus bursts (Fritze et al., 1992) which - depending on the sensitivity of the microphone used - are found in up to $74 \%$ of normals (Fritze et al., 1989). It is to be assumed that the detection rate of just $74 \%$ is dictated by the inadequacies of the equipment used (audiograms may be normal even if a larger proportion of the OHCs is lost. Lost OHCs are replaced by fibrous tissue. This tissue has different vibrating characteristics so that incessant vibrations may be intensified to the point of being recordable as SOAEs; Fritze et al., 1996).

In many recordings processed by joint timefrequency analysis chaotic distributed wavelets such as those shown in Fig. 1 are seen side by side with the sinus bursts. Like the currently known SOAEs (Ohyama et al., 1991; Ruggero et al., 1983; Hilger, 1995), these (up to now recordable) wavelets presumably reflect minor cochlear pathology. (They cannot be identified in all subjects.)

\section{THE INCESSANT VIBRATION OF OHCS}

As their vibratory frequencies suggest, $\mathrm{OHCs}$ are likely to vibrate passively (Fig. 2). Active vibrations are ruled out by the high frequencies involved $^{\dagger}$. In keeping with the chaos theory (Teich, 1995) they are bound to cluster, as already mentioned. On account of the differential resonant frequencies, energy is transported apically, i.e. towards lower resonant frequencies (Fritze, 1996). The result is a frequency difference between the vibrating and the resonant frequencies at a given site. The resultant frequency difference consumes energy so that the amplified vibration is of limited duration.

This vibration is projected into the outer ear canal. There, its frequency is equivalent to the audiofrequency of the vibrating site along the basilar membrane. Thus, incessant vibrations in terms of a chaotic sequence of wavelets of variable frequency should be expected to be present in the outer ear canal (EIV).

But even the most sensitive recording equipment (one-inch condenser microphone Bruel \& Kjaer) fails to demonstrate them when the data were processed by joint time-frequency analysis. They will presumably become detectable with the help of special algorithms for wavelet detection.

\footnotetext{
${ }^{\dagger}$ The slow motions of the OHCs probably serve as regulatory mechanisms. They may well modify the resonant frequency to the point of almost stopping vibrations (Wit et al., 1989).
} 

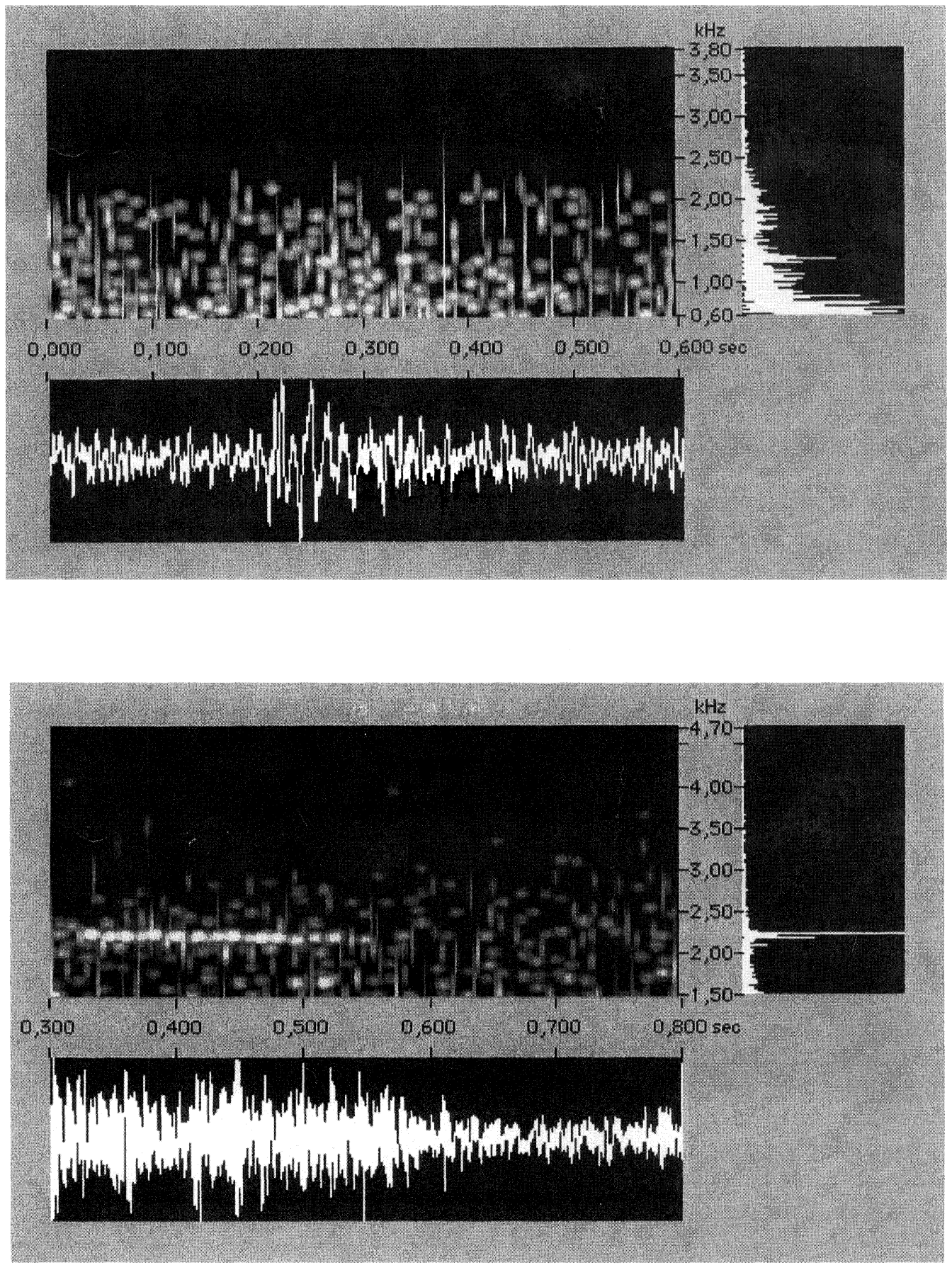

FIGURE 1 An example of oto-acoustic emissions, i.e. spontaneous emissions without any stimulus. Method: In a camera silens the microphone Bruel \& Kjaer (Naerum, Denmark) 4179 is acoustically coupled to the left ear of a 19-year-old normal. After high-pass filtering and digitalization of the signals, joint time-frequency analysis is done (adaptive spectrogram, LabVIEW ${ }^{(\mathbb{R})}$ ). The illustration shows the spectrogram (brightness-coded amplitude on the left; below it the original signal; FFT on the right). (a) The wavelets do not show any recognizable order (chaotic distribution). The vertical lines are artifacts. (b) In addition to the wavelets the first half of the trace shows a spontaneous emission. 


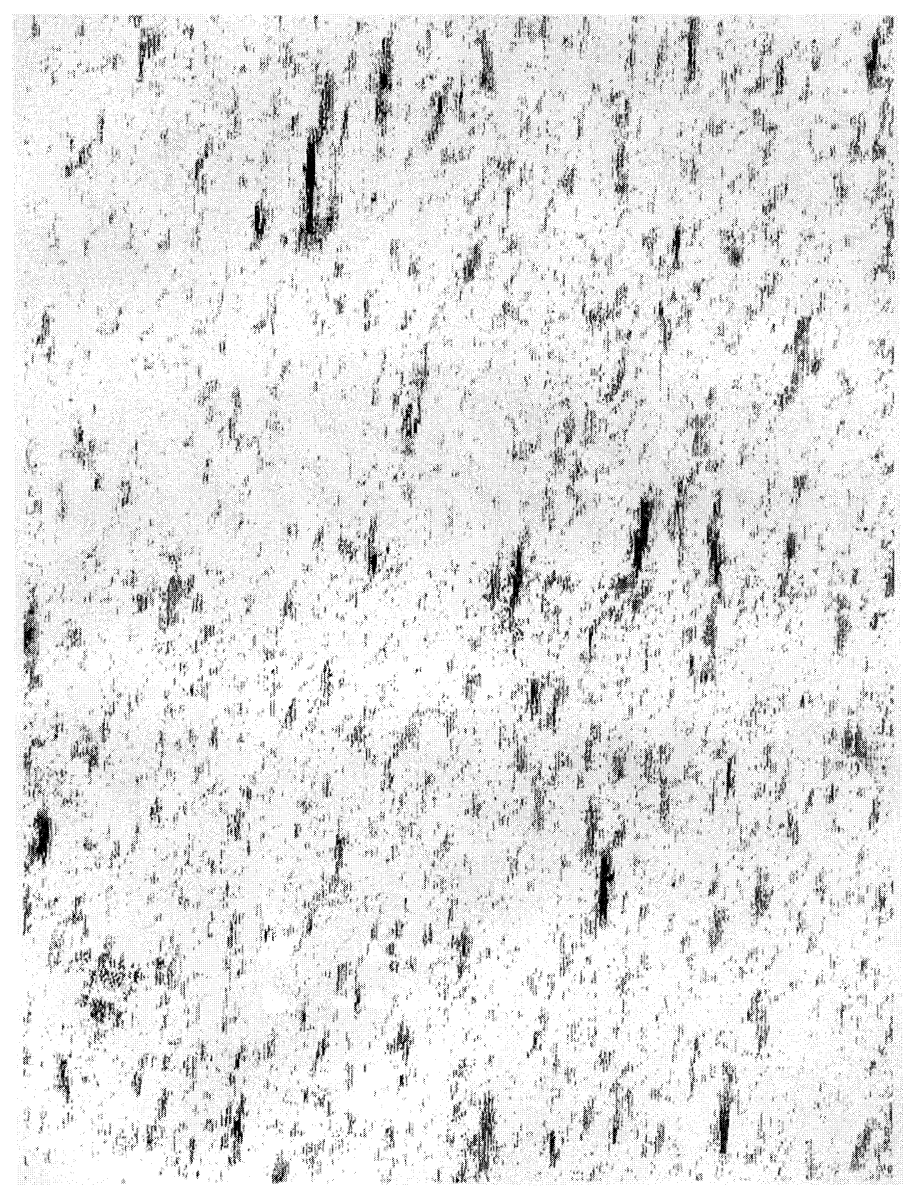

FIGURE 2 Computer simulation of likely $\mathrm{OHC}$ vibrations. Horizontal - assumed series of 640 units $(\mathrm{OHCs})$, left $=$ apical; vertical - time course (from the top down); 2 runs of the algorithm from one line to the next. The algorithm describes the reciprocal effects of coupled and randomly excited pendulums (amplitudes, phases and velocities) with the resonant frequencies rising from left to right. In the image the amplitude is shown (black = highest amplitude). Note the clustering of OHCs with a higher vibrating amplitude in terms of the chaos theory. The clusters alternate in a chaotic pattern. This should be reflected in the outer ear canal by a sequence of sinus bursts of different frequencies.

The less abnormal a cochlea, the smaller these wavelets. If they were detected in all normals, even in ears so normal that they do not produce any evoked oto-acoustic emissions recordable with standard equipment, they might well serve as a novel type of hearing test.

\section{References}

Bialek, W. (1985). Physical limits to sensation and perception. Physical Review Letters 54, 725-728.

Cohen, M. (1982). Detection threshold microstructure and its effect on temporal integration data. Journal of the Acoustic Society of America 71, 405-409.
Elliot, E. (1958). A ripple effect in the audiogram. Nature 181, 1076.

Fritze, W., Gedlicka, W. and Köhler, W. (1989). On the shape of spontaneous cochlear emissions. Il Valsalva 54, suppl. 1, $66-59$

Fritze, W., Fritze, P. and Gedlicka, W. (1992). The time course of spontaneous otoacoustic emissions. European Archiv. of Otorhinolaryngology 249, 20-23.

Fritze, W. (1996). On mechanical preprocessing in the cochlea: the three great hearing theories combined. Biochemical and Biophysical Research Communications, 223, 211-215.

Fritze, W., Steurer, M. and Fritze, P. (1996). Some ideas on the origin of otoacoustic emissions. Biochemical and Biophysical Research Communications 227, 494-498.

Henson, O.W., Koplas, P.A., Keating, A.W., Huffmann, R.F. and Henson, M.M. (1990). Cochlear resonance in the mustached bat: Behavioral adaptations. Hearing Research $\mathbf{5 0}, 259-274$. 
Hilger, A.W. (1995). The possible relationship between transient evoked otoacoustic emissions and organ of Corti irregularities in the gunea pig. Hearing Research 84, 1-11.

Kössl, M. (1994). Otoacoustic emissions from the cochleas of the 'constant frequency bats', Pteronotus parnellii and Rhinolophus rouxi. Hearing Research 72, 59-72.

Long, R. and Tubis, A. (1988). Modification of spontaneous and evoked otoacoustic emissions and associated psychoacoustic microstructure by aspirin consumption. Journal of the Acoustic Society of America 84, 1343-1353.

Ohyama, K. et al. (1985). Ultrasonic electrocochleography in guinea pig. Hearing Research 17, 143-151.

Ohyama, K. et al. (1991). Spontaneous otoacoustic emissions in the guinea pig. Hearing Research 56, 111-121.
Peat, F.D. (1987). Synchronicity, Bantam, New York.

Ruggero, M.A. et al. (1983). Spontaneous and impulsively evoked otoacoustic emissions: indicators of cochlear pathology? Hearing Research 10, 283-300.

Teich, M.C. (1995). Investigation routes to chaos in the guineapig cochlea using the continuous wavelet transform and the short-time Fourier transform. Annals of Biomedical Engineering 23, 583-607.

Wit, H.P. et al. (1989). DC injection alters spontaneous otoacoustic emission frequency in the frog. Hearing Research 41, 100-204. 


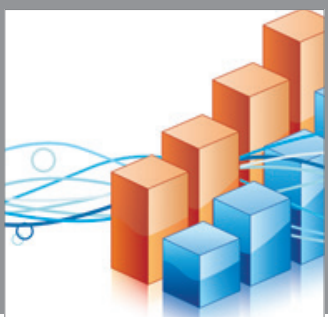

Advances in

Operations Research

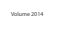

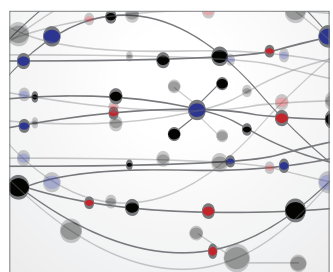

\section{The Scientific} World Journal
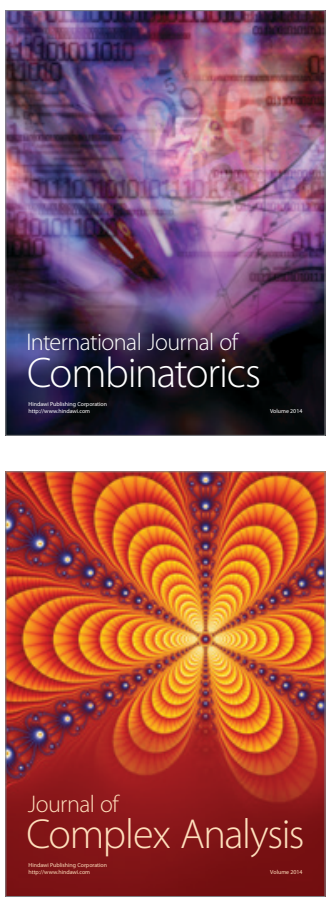

International Journal of

Mathematics and

Mathematical

Sciences
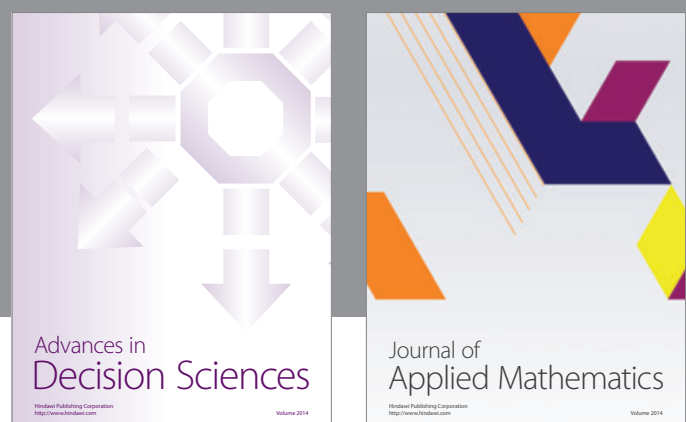

Journal of

Applied Mathematics
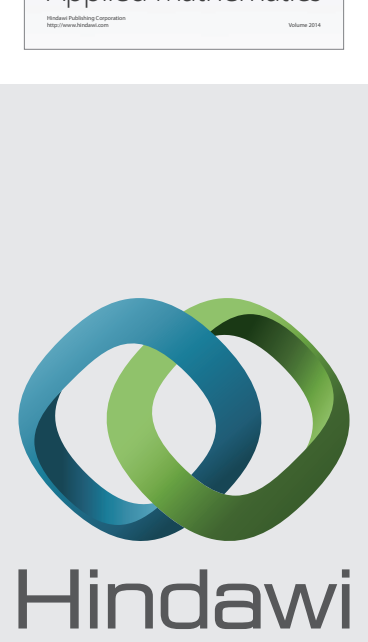

Submit your manuscripts at http://www.hindawi.com
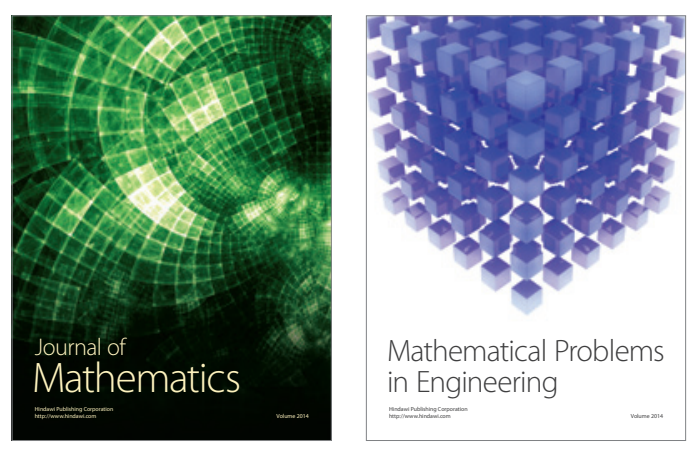

Mathematical Problems in Engineering
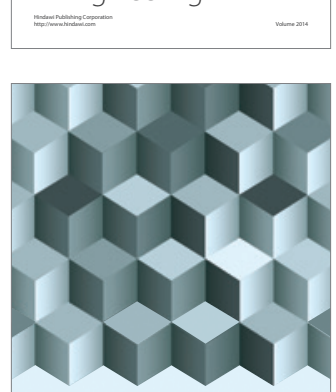

Journal of

Function Spaces
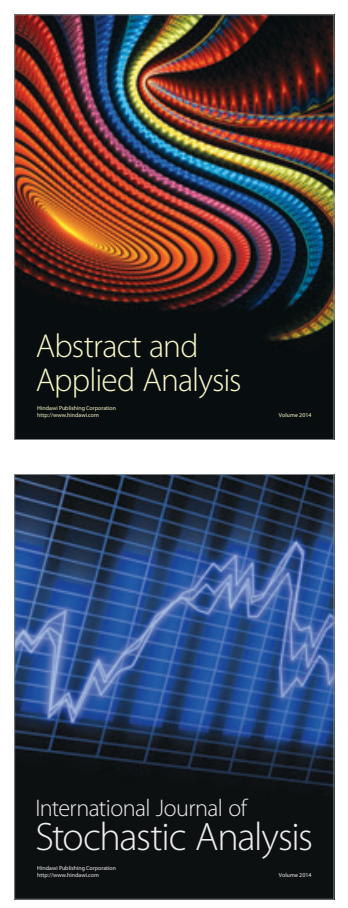

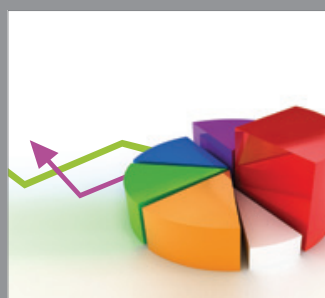

ournal of

Probability and Statistics

Promensencen
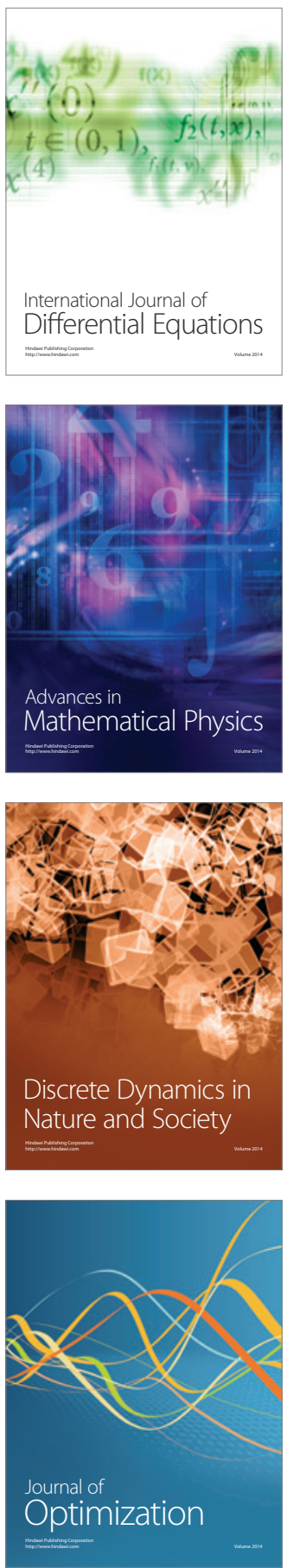